\title{
EXPLICIT MULTISTEP METHODS FOR NONSTIFF PARTIAL DIFFERENTIAL EQUATIONS
}

\author{
Panagiotis Chatzipantelidis \\ Department of Mathematics, University of Crete, 71409 Heraklion, Greece and \\ Institute of Applied and Computational Mathematics, FO.R.T.H, 71110 Heraklion, Greece.
}

\begin{abstract}
We approximate the solution of initial boundary value problems for equations of the form $A u^{\prime}(t)=B(t, u(t)), t \in\left[0, t^{\star}\right] . A$ is a linear, selfadjoint, positive definite operator on a Hilbert space $(H,(\cdot, \cdot))$ and $B$ is a possibly nonlinear operator. We discretize in space by finite element methods and for the time discretization we use explicit linear multistep schemes. We derive optimal order error estimates. The abstract results are applied to the Rosenau equation in $\mathbb{R}^{m}, m \leq 3$, to a generalized Sobolev equation in one space dimension, to a pseudoparabolic equation in $\mathbb{R}^{m}$, $m=2,3$, and to a system of equations of Boussinesq type.
\end{abstract}

\section{INTRODUCTION}

In this paper we construct and analyse explicit multistep schemes for the time discretization of a class of equations of the form: Given $t^{\star}>0$ and $u^{0} \in H$, find $u:\left[0, t^{\star}\right] \equiv J \rightarrow D(A)$ such that

$$
\begin{aligned}
A u^{\prime}(t) & =B(t, u(t)), \quad t \in J \\
u(0) & =u^{0}
\end{aligned}
$$

where $A$ is a linear, selfadjoint, positive definite operator on a Hilbert space $(H,(\cdot, \cdot))$ with domain $D(A)$ dense in $H$, and $B: J \times D(A) \rightarrow H$ a (possibly) nonlinear operator.

Let $V:=D\left(A^{1 / 2}\right)$, and define the following norm in $V,\|v\|=\left(A^{1 / 2} v, A^{1 / 2} v\right)^{1 / 2}$. Denote $|\cdot|$ the norm in $H$. We identify $H$ with its dual, let $V^{\prime}$ be the dual of $V, V \subset H \subset V^{\prime}$, and denote again by $(\cdot, \cdot)$ the duality pairing of $V^{\prime}$ and $V$. Also assume that $B$ can be extended to an operator from $J \times V$ to $V^{\prime}$. Consider now the following symmetric bilinear form $a(\cdot, \cdot): V \times V \rightarrow \mathbb{R}$ defined by

$$
a(v, w)=\left(A^{1 / 2} v, A^{1 / 2} w\right) .
$$

Using $a$ we rewrite (1.1) in the following variational form: Find $u(t) \in V, t \in J$, such that

$$
\begin{aligned}
a\left(u^{\prime}(t), v\right) & =(B(t, u(t)), v), \quad \forall v \in V, t \in J, \\
u(0) & =u^{0} .
\end{aligned}
$$

1991 Mathematics Subject Classification. Primary 65M60, 65M12; Secondary 65L06.

E-mail: chatzipa@math.uch.gr

This work was supported in part by the Greek Secretariat for Research and Technology through the PENED program, \#1747. 
Let us now consider a finite dimensional subspace $V_{h}$ of $V$. The corresponding semidiscrete problem will be: Find a function $u_{h}, u_{h}(t) \in V_{h}, t \in J$, such that

$$
\begin{aligned}
a\left(u_{h}^{\prime}(t), \chi\right) & =\left(B\left(t, u_{h}(t)\right), \chi\right), \quad \forall \chi \in V_{h}, t \in J, \\
u_{h}(0) & =u_{h}^{0},
\end{aligned}
$$

with $u_{h}^{0} \in V_{h}$ a given approximation to $u^{0}$. In order to construct fully discrete approximations to $u$ we have to discretize (1.3) in time. We do this using explicit multistep schemes. Thus consider a uniform partition of $J$ with time step $k:=\frac{t^{\star}}{N}, N \in \mathbb{N}$, and time levels $t^{n}:=n k$, $n=0, \ldots, N$. A linear explicit multistep scheme is characterized by two polynomials $\alpha, \beta$,

$$
\alpha(z)=\sum_{j=0}^{q} \alpha_{j} z^{j}, \quad \beta(z)=\sum_{j=0}^{q-1} \beta_{j} z^{j} .
$$

Based on (1.3) and an explicit multistep scheme we define approximations $U^{n}, U^{n} \in V_{h}$, to $u^{n}=u\left(t^{n}\right)$ by

$$
\sum_{j=0}^{q} \alpha_{j} a\left(U^{n+j}, \chi\right)=k \sum_{j=0}^{q-1} \beta_{j}\left(B\left(t^{n+j}, U^{n+j}\right), \chi\right), \quad \forall \chi \in V_{h} .
$$

We shall assume that the linear multistep method, defined by the polynomials $\alpha$ and $\beta$, we use to discretize (1.3) in time, is stable and consistent. In $\S 2$ we consider a problem equivalent to (1.2): Find $u(t) \in V, t \in J$, such that

$$
\begin{aligned}
\left(u^{\prime}(t), v\right) & =(T B(t, u(t)), v), \quad \forall v \in V, t \in J, \\
u(0) & =u^{0}
\end{aligned}
$$

$T$ denoting the solution operator of the problem: Given $f \in V^{\prime}$, find $v \in V$, such that

$$
a(v, w)=(f, w), \quad \forall w \in V .
$$

We further assume that the operators $B$ and $T B$ satisfy a "Lipschitz"-like property, cf. (H3) and (H4) in $\S 2$. Due to this assumption, we do not have to impose restrictive mesh conditions or use a time discretization method with good stability properties, for example, Astability, for the resulting fully discrete scheme to be stable. Given $U^{n+j} \in V_{h}, j=0, \ldots, q-1$, we calculate $U^{n+q} \in V_{h}$ by solving an equivalent to scheme (1.5) linear system of dimension $m=\operatorname{dim} V_{h}$. This system has a unique solution due to the properties of operator $A$. Therefore, given $U^{0}, \ldots, U^{q-1} \in V_{h}, U^{q}, \ldots, U^{N}$ are well defined. Their calculation is not expensive because at every time level we solve a linear system with the same matrix.

An alternative to multistep methods are Runge-Kutta methods which are widely used because of their good stability propetries. If we would use an explicit $q$-stage Runge-Kutta method instead, at every time step, we would have to solve $q+1$ linear systems of dimension $m$. Also, we consider explicit methods since in the case of an implicit one we would have to solve a (possibly) nonlinear system of dimension $m$ in the case of multistep methods, and of dimension $q m$ in the case of a $q$-stage Runge-Kutta method at every time level.

Throughout this paper we assume that problem (1.1) has a unique and sufficiently smooth solution $u$. A brief overview is the following: In $\S 2$ we introduce an "elliptic" projection operator 
to $V_{h}$ and we assume that $V_{h}$ satisfies two approximation properties, (H1) and (H2). That is, we can approximate every function of $V$, from $V_{h}$, with order $d$ in $|\cdot|$, and further, every smooth function with order $r$ in $|\cdot|$ and $r-d$ in $\|\cdot\|$, with integers $r$, $d$ satisfying, $0<d<r$. In Lemma 2.1, we prove optimal error estimates of the time derivative of order $\ell, \ell=0,1, \ldots$, of the difference of the solution $u$ of (1.1) with its "elliptic" projection in the norms $|\cdot|$ and $\|\cdot\|$. We also assume hypotheses (H3) and (H4) for operators $B$ and $T B$, respectively. In $\S 3$ we study a simple, explicit, one step scheme for the discretization in time of (1.3), the explicit Euler. We do this as an introduction to the analysis of explicit multistep methods. In $\S 4$ we consider an explicit multistep method of order $p$. Then, for suitable initial approximations $U^{0}, \ldots, U^{q-1}$ and $k, h$ sufficiently small, we prove

$$
\max _{0 \leq n \leq N}\left|u\left(t^{n}\right)-U^{n}\right| \leq C\left(k^{p}+h^{r}\right) \quad \text { and } \max _{0 \leq n \leq N}\left\|u\left(t^{n}\right)-U^{n}\right\| \leq C\left(k^{p}+h^{r-d}\right),
$$

with a constant $C$ independent of $k$ and $h$. Finally, in $\S 5$, we apply this result to four specific examples, to the Rosenau equation, to a generalized Sobolev type equation, to a pseudoparabolic equation and to a Boussinesq type system of equations. This paper is motivated by the recent work of Akrivis, Crouzeix and Makridakis, [1], on implicit-explicit multistep methods for nonlinear parabolic problems.

\section{Preliminaries}

We consider a family $\left\{V_{h}\right\}_{0<h \leq 1}$ of finite dimensional subspaces of $V$. Using the auxiliary problem (1.7) we will state equivalent problems to (1.2) and (1.3) that will help us analyse the method (1.5) in $\S 3$ and $\S 4$. We will also assume some properties for the finite dimensional space $V_{h}$ and the operators $B$ and $T B$.

Thus, consider problem (1.7). Obviously, $a(\cdot, \cdot)$ is an inner product in $V$. Also $(V, a(\cdot, \cdot))$ is a Hilbert space, continuously embedded in $H$, (see, e.g., [8, Chapter VIII, §3, Theorem 13]). Therefore, the solution $v$ of problem (1.7) is well defined. Denote by $T, T: V^{\prime} \rightarrow V, T f=v$, the solution operator of this problem; then

$$
a(T f, w)=(f, w), \quad \forall w \in V .
$$

Choosing now $w=T f$ in (2.1) we easily obtain,

$$
\|T f\| \leq\|f\|_{\star}
$$

$\|\cdot\|_{\star}$ denoting the dual norm of $V$. In the sequel, we consider the discrete analogue of (2.1). For $f \in V^{\prime}$, find $v_{h} \in V_{h}$, such that

$$
a\left(v_{h}, \chi\right)=(f, \chi), \quad \forall \chi \in V_{h}
$$

This problem is equivalent to a linear system with square matrix and it is easily seen that it has a unique solution. Denote by $T_{h}: V^{\prime} \rightarrow V_{h}, T_{h} f=v_{h}$, the solution operator of the above problem; then

$$
a\left(T_{h} f, \chi\right)=\underset{3}{(f, \chi),} \quad \forall \chi \in V_{h} .
$$


Also in view of (2.3) and the symmetry of the bilinear form $a$, we obtain

$$
\left(T_{h} f, g\right)=a\left(T_{h} f, T_{h} g\right)=\left(f, T_{h} g\right), \quad \forall f, g \in V^{\prime} .
$$

In the sequel we will use the "elliptic" projection operator to $V_{h}, R_{h}: V \rightarrow V_{h}$, defined by

$$
a\left(R_{h} v, \chi\right)=a(v, \chi), \quad \forall \chi \in V_{h} .
$$

It is easily seen that $R_{h}$ is well defined. For the error analysis that will follow we will need some approximation properties for the "elliptic" projection $R_{h}$. Therefore we assume the following:

Hypothesis 1: There exists a constant $C$, such that

$$
\left|v-R_{h} v\right| \leq C h^{d}\|v\|, \quad \forall v \in V
$$

with an integer $d>0$.

Supposing that $v$ is sufficiently smooth we further assume:

Hypothesis 2: $R_{h} v$ is an approximation to $v$ of order $r$,

$$
\left|v-R_{h} v\right|+h^{d}\left\|v-R_{h} v\right\| \leq N(v) h^{r},
$$

with $N(v)$ bounded, if a suitable norm of $v$ is bounded, and an integer $r, r>d$.

Using (2.5), (2.3) and (2.1), we obtain $T_{h}=R_{h} T$. Therefore, in view of (H1), we easily see that $T_{h} f$ is an approximation to $T f$, of order $d$,

$$
\left|T f-T_{h} f\right| \leq C h^{d}\|T f\|, \quad \forall f \in V^{\prime} .
$$

Having defined operators $T$ and $T_{h}$, we can state problem (1.2) equivalently as (1.6), and (1.3) as: Find $u_{h}(t) \in V_{h}, t \in J$, such that

$$
\begin{aligned}
\left(u_{h}^{\prime}(t), \chi\right) & =\left(T_{h} B\left(t, u_{h}(t)\right), \chi\right), \quad \forall \chi \in V_{h}, t \in J, \\
u_{h}(0) & =u_{h}^{0},
\end{aligned}
$$

with $u_{h}^{0} \in V_{h}$ an approximation to $u^{0}$.

In the sequel, let $W(t):=R_{h} u(t)$ and $\varrho(t):=u(t)-W(t), t \in J$. Also, if $\varphi$ is a function defined in the interval $J$, denote $\frac{d^{\ell}}{d t^{\ell}} \varphi$ by $\varphi^{(\ell)}$. Because the bilinear form $a$ is independent of $t$, from (H2) and the definition of the projection $R_{h},(2.5)$, we have

Lemma 2.1: Let $u$ be a sufficiently smooth solution of (1.1). Then there exist constants $C_{\ell}\left(N\left(u^{(\ell)}\right)\right), \ell=0,1, \ldots$, independent of $h$, such that

$$
\max _{t \in J}\left|\varrho^{(\ell)}(t)\right| \leq C_{\ell} h^{r}, \quad \max _{t \in J}\left\|\varrho^{(\ell)}(t)\right\| \leq C_{\ell} h^{r-d}, \quad \ell=0,1, \ldots
$$

Let now $M=\{v \in V: \exists t \in J,\|u(t)-v\|<1\}$ and assume for the operators $B$ and $T B$ :

Hypothesis 3: There exists a constant $L_{1}$, independent of $t$ and $h$, such that

$$
\|B(t, v)-B(t, W(t))\|_{\star} \leq L_{1}\|v-W(t)\|, \quad \forall v \in M, \forall t \in J .
$$


Hypothesis 4: There exists a constant $L_{2}$, independent of $t$ and $h$, such that

$$
|T B(t, v)-T B(t, W(t))| \leq L_{2}|v-W(t)|, \quad \forall v \in M, \forall t \in J
$$

Using then (2.6), (H4), (2.2) and (H3), we can prove

$$
\left|T_{h} B(t, v)-T_{h} B(t, W(t))\right| \leq L\left(|v-W(t)|+h^{d}\|v-W(t)\|\right), \quad \forall v \in M,
$$

with a constant $L$, independent of $t$ and $h$. Choosing now $v=u(t)$ in (2.9) and using (2.8), we obtain

$$
\left|T_{h} B(t, u(t))-T_{h} B(t, W(t))\right| \leq C h^{r},
$$

with a constant $C(N(u))$ independent of $t$ and $h$.

Remark 2.1: The assumption $V_{h} \subset V$ is not essential in our analysis. One can use an approximation space $V_{h} \nsubseteq V$ by appropriately modifying the definition of the discrete operator $R_{h}$, the set $M$ and (H3) and (H4).

\section{Fully discrete SChemes: Explicit Euler Method}

In this section we will study the simplest method for the discretization in time of (1.3), the explicit Euler scheme. In the next section we will generalize our results for explicit multistep schemes.

Consider a uniform partition of the interval $J$. For $N \in \mathbb{N}$, let $k=\frac{t^{\star}}{N}$ be the time step and $t^{n}:=n k, n=0, \ldots, N$, the time levels. Also for a function $\varphi$, defined on the interval $J$, let $\varphi^{n}:=\varphi\left(t^{n}\right)$ and for $v^{0}, \ldots, v^{N} \in V_{h}$, let $\partial v^{n}:=\frac{v^{n+1}-v^{n}}{k}, n=0, \ldots, N-1$.

We discretize (1.3) in time by the explicit Euler method. The resulting scheme is

$$
\begin{aligned}
a\left(\partial U^{n}, \chi\right) & =\left(B\left(t^{n}, U^{n}\right), \chi\right), \quad \forall \chi \in V_{h}, n=0, \ldots, N-1, \\
U^{0} & =u_{h}^{0},
\end{aligned}
$$

where $u_{h}^{0} \in V_{h}$ satisfies

$$
\left|u^{0}-u_{h}^{0}\right|+h^{d}\left\|u^{0}-u_{h}^{0}\right\| \leq C h^{r} .
$$

The approximations $U^{n}, n=0, \ldots, N$, given by (3.1) are well defined, because given $U^{n} \in V_{h}$, $U^{n+1}$ is the unique solution of a linear system with symmetric and positive definite matrix, due to the properties of operator $A$. Our objective is to estimate the approximation error $u^{n}-U^{n}, n=0, \ldots, N$. As an auxiliary result we will first show consistency of scheme (3.1) for the "elliptic" projection $W$ of the solution $u$ of (1.1). The consistency error $E^{n}$ of the scheme (3.1) for $W$ is given by

$$
E^{n}=\partial W^{n}-T_{h} B\left(t^{n}, W^{n}\right), \quad n=0, \ldots, N-1 .
$$

Using now (3.3), (2.5), (2.3) and (1.2), for $\chi \in V_{h}$, we have

$$
k a\left(E^{n}, \chi\right)=a\left(u^{n+1}-u^{n}-k u^{\prime}\left(t^{n}\right), \chi\right)+k\left(B\left(t^{n}, u^{n}\right)-B\left(t^{n}, W^{n}\right), \chi\right) .
$$


Therefore, using (H3) and choosing $\chi=E^{n}$ in (3.4), we obtain

$$
\max _{0 \leq n \leq N-1}\left\|E^{n}\right\| \leq C_{\star}\left(k+h^{r-d}\right),
$$

with a constant $C_{\star}\left(\left\|u^{(2)}\right\|, N(u)\right)$ independent of $k$ and $h$. Next, choosing $\chi=T_{h} E^{n}$ in (3.4), in view of the symmetry of the bilinear form $a,(2.3),(1.2)$, and (2.4), we can easily obtain, for $n=0, \ldots, N-1$,

$$
\begin{aligned}
k a\left(E^{n}, T_{h} E^{n}\right)= & -\left(\varrho^{n+1}-\varrho^{n}-k \varrho^{\prime}\left(t^{n}\right), E^{n}\right)+\left(u^{n+1}-u^{n}-k u^{\prime}\left(t^{n}\right), E^{n}\right) \\
& +k\left(T_{h} B\left(t^{n}, u^{n}\right)-T_{h} B\left(t^{n}, W^{n}\right), E^{n}\right) .
\end{aligned}
$$

Hence, due to (2.8) and (2.10), we get

$$
\max _{0 \leq n \leq N-1}\left|E^{n}\right| \leq C\left(k+h^{r}\right)
$$

with a constant $C\left(\left|u^{(2)}\right|, N(u), N\left(u^{(2)}\right)\right)$ independent of $k$ and $h$.

In the sequel, using the consistency error estimations for the "elliptic" projection $W$, we will prove the main result of this paragraph, the convergence of the scheme (3.1).

Theorem 3.1 Assume that the solution $u$ of problem (1.1) is sufficiently smooth, and $h$ and $k$ are sufficiently small. Then, there exists a constant $C\left(\left\|u^{(2)}\right\|, N(u), N\left(u^{(2)}\right)\right)$, independent of $h$ and $k$, such that

$$
\max _{0 \leq n \leq N}\left\|u^{n}-U^{n}\right\| \leq C\left(k+h^{r-d}\right) \quad \text { and } \max _{0 \leq n \leq N}\left|u^{n}-U^{n}\right| \leq C\left(k+h^{r}\right) .
$$

Proof: Let $\varrho^{n}:=u^{n}-W^{n}$ and $\vartheta^{n}:=W^{n}-U^{n}, n=0, \ldots, N$. Then $u^{n}-U^{n}=\varrho^{n}+\vartheta^{n}$. In order to show (3.7) we will estimate $\varrho^{n}$ and $\vartheta^{n}$ in the corresponding norms. From (2.8) we easily obtain

$$
\max _{0 \leq n \leq N}\left(\left|\varrho^{n}\right|+h^{d}\left\|\varrho^{n}\right\|\right) \leq C h^{r} .
$$

Hence, we only have to estimate $\vartheta^{n}$. According to (3.2) and (3.8), there exists a constant $C_{\star \star}$, independent of $h$, such that

$$
\left|\vartheta^{0}\right|+h^{d}\left\|\vartheta^{0}\right\| \leq C_{\star \star} h^{r} .
$$

From (3.1), (3.3) and (2.3), we get the following error equation, for $\chi \in V_{h}$ and $n=0, \ldots, N-1$,

$$
a\left(\vartheta^{n+1}, \chi\right)=a\left(\vartheta^{n}, \chi\right)+k\left(B\left(t^{n}, W^{n}\right)-B\left(t^{n}, U^{n}\right), \chi\right)+k a\left(E^{n}, \chi\right) .
$$

Let now $L_{1}$ be the "Lipschitz" constant in (H3), $C_{\star}$ and $C_{\star \star}$ the constants in (3.5) and (3.9), respectively, and $c:=\max \left(1+L_{1}, C_{\star}, C_{\star \star}\right)$. We will show, by induction over $n$, that

$$
\left\|\vartheta^{n}\right\| \leq c_{n}\left(k+h^{r-d}\right), \quad \text { with } \quad c_{n}=c(1+c k)^{n}, \quad n=0, \ldots, N .
$$

Obviously, there exists a constant $\widetilde{C}$, independent of $k$ and $h$, such that

$$
\max _{0 \leq n \leq N} c_{n} \leq \widetilde{C}
$$


Since $r>d$, suppose now that $h$ and $k$ are sufficiently small, such that

$$
C h^{r}+\widetilde{C}\left(k+h^{r-d}\right)<1,
$$

with $\widetilde{C}$ and $C$ the constants in (3.12) and (3.8), respectively. According to (3.9), (3.11) holds for $n=0$. Next, assume that (3.11) holds for $n, 0 \leq n \leq N-1$. From the induction hypothesis, (3.8) and (3.13), we obtain

$$
U^{n} \in M
$$

Choosing now $\chi=\vartheta^{n+1}$ in the error equation (3.10), in view of (3.14), (H3) and the induction hypothesis, we can show

$$
\left\|\vartheta^{n+1}\right\| \leq\left(1+L_{1} k\right)\left\|\vartheta^{n}\right\|+k\left\|E^{n}\right\| \leq c(1+c k)^{n+1}\left(k+h^{r-d}\right) .
$$

Therefore, (3.11) holds for $n+1$, and so it holds for $n=0, \ldots, N$. Hence, from (3.12), we obtain

$$
\left\|\vartheta^{n}\right\| \leq \widetilde{C}\left(k+h^{r-d}\right), \quad n=0, \ldots, N .
$$

If we now choose $\chi=T_{h} \vartheta^{n+1}$ in the error equation (3.10), in view of the symmetry of the bilinear form $a,(2.3)$ and (2.4), we get

$$
\left(\vartheta^{n+1}, \vartheta^{n+1}\right)=\left(\vartheta^{n}, \vartheta^{n+1}\right)+k\left(T_{h}\left(B\left(t^{n}, W^{n}\right)-B\left(t^{n}, U^{n}\right)\right), \vartheta^{n+1}\right)+k\left(E^{n}, \vartheta^{n+1}\right) .
$$

Using then (3.14), (2.9), (3.16) and (3.6) in the previous relation, we have, for $n=0, \ldots N-1$,

$$
\left|\vartheta^{n+1}\right| \leq(1+L k)\left|\vartheta^{n}\right|+L k h^{d}\left\|\vartheta^{n}\right\|+k\left|E^{n}\right| \leq(1+L k)\left|\vartheta^{n}\right|+C k\left(k+h^{r}\right) .
$$

Therefore, from (3.17), (3.9) and the discrete Gronwall lemma, we obtain

$$
\max _{0 \leq n \leq N}\left|\vartheta^{n}\right| \leq C\left(k+h^{r}\right) .
$$

The estimate (3.7) follows immediately from (3.18), (3.16) and (3.8).

\section{Fully DiscRete SChemes: ExPlicit MultisteP Methods}

We shall next analyse fully discrete schemes for the discretization of (1.1), obtained by discretizing (1.3) in time with explicit $q$-step methods. In Theorem 4.1 we show optimal order of convergence in the norms $\|\cdot\|$ and $|\cdot|$.

Consider an explicit $q$-step method and let $\alpha, \beta$ be the two polynomials, see (1.4), that characterize it. We assume that the multistep method satisfies the root condition:

$$
\begin{aligned}
& \text { If } z_{0} \text { is a root of } \alpha \text {, then }\left|z_{0}\right| \leq 1 \text {. } \\
& \text { If } z_{0} \text { is a multiple root of } \alpha \text {, then }\left|z_{0}\right|<1 \text {. }
\end{aligned}
$$

Also assume that it has order $p \in \mathbb{N}$ :

$$
C_{j}=0, \quad j=0, \ldots, p, \quad C_{p+1} \neq 0,
$$


with

$$
C_{0}=\alpha(1), \quad C_{j}=\frac{1}{i !}\left(\sum_{j=0}^{q} \alpha_{j} j^{i}-i \sum_{j=0}^{q-1} \beta_{j} j^{i-1}\right), \quad i \geq 1 .
$$

Several explicit multistep methods satisfy these stability and consistency conditions, see, e.g., [11, Chapter III.1]. Conditions (4.1) and (4.2) yield the consistency property (cf., e.g., [12, $\S 5.2-5])$ :

$$
\max _{0 \leq n \leq N-q}\left\|\left|\sum_{j=0}^{q} \alpha_{j} v^{n+j}-k \sum_{j=0}^{q-1} \beta_{j} v^{\prime}\left(t^{n+j}\right)\left\|\mid \leq C k^{p+1} \max _{t \in J}\right\| v^{(p+1)}(t)\|\|,\right.\right.
$$

for every function $v \in C^{p+1}(J ; V)$, where by $\|\mid\| \|$, we denote either norm $|\cdot|$ or $\|\cdot\|$. Further, assume we are given approximations $U^{0}, \ldots, U^{q-1} \in V_{h}$ to $u^{0}, \ldots, u^{q-1}$, such that

$$
\sum_{j=0}^{q-1}\left|u^{j}-U^{j}\right| \leq C\left(k^{p}+h^{r}\right) \quad \text { and } \quad \sum_{j=0}^{q-1}\left\|u^{j}-U^{j}\right\| \leq C\left(k^{p}+h^{r-d}\right) .
$$

We define now approximations $U^{n} \in V_{h}$ to $u^{n}, q \leq n \leq N$, by

$$
\sum_{j=0}^{q} \alpha_{j} a\left(U^{n+j}, \chi\right)=k \sum_{j=0}^{q-1} \beta_{j}\left(B\left(t^{n+j}, U^{n+j}\right), \chi\right), \quad \forall \chi \in V_{h}, n=0, \ldots, N-q .
$$

The approximations $U^{n}, n=q, \ldots, N$, are well defined, because given $U^{n}, \ldots, U^{n+q-1} \in V_{h}$, $U^{n+q}$ is the unique solution of a linear system with symmetric and positive definite matrix, due to the properties of operator $A$. In the sequel we will estimate the difference $u^{n}-U^{n}, n=0, \ldots, N$. As an intermediate step we will show consistency of scheme (4.5) for the "elliptic" projection $W$ of the solution $u$ of (1.1).

The consistency error $E^{n}$ of scheme (4.5) for $W$ is given by

$$
k E^{n}=\sum_{j=0}^{q} \alpha_{j} W^{n+j}-k \sum_{j=0}^{q-1} \beta_{j} T_{h} B\left(t^{n+j}, W^{n+j}\right), \quad n=0, \ldots, N-q .
$$

Using (4.6), (2.5), (2.3) and (1.2), for $\chi \in V_{h}, n=0, \ldots, N-q$, it is easily seen

$$
\begin{aligned}
k a\left(E^{n}, \chi\right)= & a\left(\sum_{j=0}^{q} \alpha_{j} u^{n+j}-k \sum_{j=0}^{q-1} \beta_{j} u^{\prime}\left(t^{n+j}\right), \chi\right) \\
& +k \sum_{j=0}^{q-1} \beta_{j}\left(B\left(t^{n+j}, u^{n+j}\right)-B\left(t^{n+j}, W^{n+j}\right), \chi\right) .
\end{aligned}
$$

Choosing now $\chi=E^{n}$ in the previous relationship, due to (4.3) and (H3), for $n=0, \ldots, N-q$, we get

$$
\max _{0 \leq n \leq N-q}\left\|E^{n}\right\| \leq D_{\star}\left(k^{p}+h^{r-d}\right),
$$


with a constant $D_{\star}\left(\left\|u^{(p+1)}\right\|, N(u)\right)$ independent of $k$ and $h$. Also choosing $\chi=T_{h} E^{n}$ in (4.7), in view of the symmetry of the bilinear form $a$, and (2.5), (1.2) and (2.4), for $n=0, \ldots, N-q$, we obtain

$$
\begin{aligned}
& k a\left(E^{n}, T_{h} E^{n}\right)=-\left(\sum_{j=0}^{q} \alpha_{j} \varrho^{n+j}-k \sum_{j=0}^{q-1} \beta_{j} \varrho^{\prime}\left(t^{n+j}\right), E^{n}\right)+\sum_{j=0}^{q} \alpha_{j}\left(u^{n+j}, E^{n}\right) \\
& \quad-k \sum_{j=0}^{q-1} \beta_{j}\left(u^{\prime}\left(t^{n+j}\right), E^{n}\right)+k \sum_{j=0}^{q-1} \beta_{j}\left(T_{h} B\left(t^{n+j}, u^{n+j}\right)-T_{h} B\left(t^{n+j}, W^{n+j}\right), E^{n}\right) .
\end{aligned}
$$

Using next (4.3), (2.8) and (2.10), for $n=0, \ldots, N-q$, in the previous relation, we get

$$
\max _{0 \leq n \leq N-q}\left|E^{n}\right| \leq C\left(k^{p}+h^{r}\right),
$$

with a constant $C\left(\left|u^{(p+1)}\right|, N(u), N\left(u^{(p+1)}\right)\right)$ independent of $k$ and $h$. Using the consistency error estimations for the "elliptic" projection $W$, we will prove the main result of this section, Theorem 4.1, the convergence of method (4.5). In the sequel we shall use the notation

$$
\begin{gathered}
\Theta^{n}=\left(\begin{array}{c}
\vartheta^{n+q-1} \\
\vdots \\
\vartheta^{n}
\end{array}\right), \quad \mathcal{E}^{n}=\left(\begin{array}{c}
E^{n} \\
0 \\
\vdots \\
0
\end{array}\right), \quad \Gamma_{n}^{j}=\beta_{j}^{\prime}\left(B\left(t^{n+j}, W^{n+j}\right)-B\left(t^{n+j}, U^{n+j}\right)\right), \\
\Lambda=\left(\begin{array}{cccc}
\alpha_{q-1}^{\prime} & \alpha_{q-2}^{\prime} & \ldots & \alpha_{0}^{\prime} \\
1 & 0 & \ldots & 0 \\
& \ddots & \ddots & \\
0 & \ldots & 1 & 0
\end{array}\right), \quad \Gamma_{n}=\left(\begin{array}{ccc}
\Gamma_{n}^{q-1} & \ldots & \Gamma_{n}^{0} \\
0 & \ldots & 0 \\
\vdots & & \vdots \\
0 & \ldots & 0
\end{array}\right),
\end{gathered}
$$

where $\alpha_{j}^{\prime}=\frac{\alpha_{j}}{\alpha_{q}}, \beta_{j}^{\prime}=\frac{\beta_{j}}{\alpha_{q}}, j=0, \ldots, q-1$. Further, for $V=\left(v_{1}, \ldots, v_{q}\right)^{T}$ and $W=\left(w_{1}, \ldots, w_{q}\right)^{T}$ in $H^{q}$ or in $V^{q}$,

$$
\begin{gathered}
(V, W):=\sum_{i=1}^{q}\left(v_{i}, w_{i}\right), \quad|V|:=\left(\sum_{i=1}^{q}\left|v_{i}\right|^{2}\right)^{1 / 2}, \quad\|V\|:=\left(\sum_{i=1}^{q}\left\|v_{i}\right\|^{2}\right)^{1 / 2}, \\
\|V\|_{\star}:=\left(\sum_{i=1}^{q}\left\|v_{i}\right\|_{\star}^{2}\right)^{1 / 2}, \quad a(V, W):=\sum_{i=1}^{q} a\left(v_{i}, w_{i}\right), \quad T_{h} V=\left(T_{h} v_{1}, \ldots, T_{h} v_{q}\right)^{T} .
\end{gathered}
$$

Then, there exists an invertible matrix $S$ such that the subordinate Euclidean matrix norm $\|\cdot\|_{2}$ of the matrix $\mathcal{L}=S^{-1} \Lambda S$ is bounded by one,

$$
\|\mathcal{L}\|_{2} \leq 1 \text {. }
$$

For the corresponding result in the subordinate matrix norm $\|\cdot\|_{\infty}$ of $\mathbb{R}^{q}$, cf., e.g., $[11$, Chapter III, Lemma 4.4]. Relation (4.10) follows by similar arguments.

Theorem 4.1: Let $\left\{U^{n}\right\}_{n=0}^{N}$ satisfy (4.5), let (4.1), (4.2) and (4.4) hold, and the solution u of (1.1) be sufficiently smooth. Then, for $h$ and $k$ sufficiently small, there exists a constant $C\left(\left\|u^{(p+1)}\right\|, N(u), N\left(u^{(p+1)}\right)\right)$, independent of $k$ and $h$, such that

$$
\max _{0 \leq n \leq N}\left\|u^{n}-U^{n}\right\| \leq C\left(k^{p}+h^{r-d}\right) \quad \text { and } \max _{0 \leq n \leq N}\left|u^{n}-U^{n}\right| \leq C\left(k^{p}+h^{r}\right) .
$$


Proof: Let $\varrho^{n}$ and $\vartheta^{n}$ be as in the proof of Theorem 3.1. Then, according to (3.8), it suffices to estimate $\vartheta^{n}$. From (4.5) and (4.6), for $\chi \in V_{h}, n=0, \ldots, N-q$, we obtain

$$
\sum_{j=0}^{q} \alpha_{j} a\left(\vartheta^{n+j}, \chi\right)=k a\left(E^{n}, \chi\right)+k \sum_{j=0}^{q-1} \beta_{j}\left(B\left(t^{n+j}, W^{n+j}\right)-B\left(t^{n+j}, U^{n+j}\right), \chi\right) .
$$

Next, let $Y^{n}=S^{-1} \Theta^{n}, \widetilde{\Gamma_{n}}=S^{-1} \Gamma_{n}, \widetilde{\mathcal{E}^{n}}=S^{-1} \mathcal{E}^{n}$ and $e=(1, \ldots, 1)^{T}$. Instead of estimating $\vartheta^{n}$ we will estimate $Y^{n}$. For this reason we rewrite (4.12) as

$$
a\left(Y^{n+1}, X\right)=a\left(\mathcal{L} Y^{n}, X\right)+k\left(\widetilde{\Gamma_{n}} e, X\right)+k \alpha_{q}^{-1} a\left(\widetilde{\mathcal{E}^{n}}, X\right), \quad \forall X \in V^{q}, n=0, \ldots, N-q
$$

According to (4.4) and (3.8), we can easily see that

$$
\left\|Y^{0}\right\| \leq\left\|S^{-1}\right\|_{2}\left\|\Theta^{0}\right\| \leq D_{\star \star}\left(k^{p}+h^{r-d}\right) \quad \text { and } \quad\left|Y^{0}\right| \leq\left\|S^{-1}\right\|_{2}\left|\Theta^{0}\right| \leq D_{\star \star}\left(k^{p}+h^{r}\right)
$$

with a constant $D_{\star \star}$ independent of $k$ and $h$. Let now $L_{1}$ be the "Lipschitz" constant in (H3), $D_{\star}$ and $D_{\star \star}$ the constants in (4.8) and (4.14), respectively, and $c:=\max \left(1+\max _{j}\left|\beta_{j}^{\prime}\right|\left\|S^{-1}\right\|_{2} L_{1}\right.$, $\left.D_{\star} \alpha_{q}^{-1}\left\|S^{-1}\right\|_{2}, D_{\star \star}\right)$. In the sequel we will show, by induction over $n$, that

$$
\left\|Y^{n}\right\| \leq d_{n}\left(k^{p}+h^{r-d}\right), \quad \text { with } \quad d_{n}=c(1+c k)^{n}, \quad n=0, \ldots, N-q+1 .
$$

Obviously, there exists a constant $\widetilde{D}$, independent of $k$ and $h$, such that

$$
\max _{0 \leq n \leq N-q+1} d_{n} \leq \widetilde{D}
$$

Since $r>d$, suppose now that $h$ and $k$ are sufficiently small, such that

$$
C h^{r}+\widetilde{D}\left(k^{p}+h^{r-d}\right)<1,
$$

with $\widetilde{D}$ and $C$ the constants in (4.16) and (3.8), respectively. According to (4.14), (4.15) holds for $n=0$. Next, assume that (4.15) holds for $n, 0 \leq n \leq N-q$. From the induction hypothesis, (3.8) and (4.17), we get

$$
U^{n} \in M
$$

Choosing $X=Y^{n+1}$ in the error equation (4.13), in view of (4.18), (H3) and the induction hypothesis, we obtain

$$
\left\|Y^{n+1}\right\| \leq\left(1+\max _{j}\left|\beta_{j}^{\prime}\right| L_{1}\left\|S^{-1}\right\|_{2} k\right)\left\|Y^{n}\right\|+k \alpha_{q}^{-1}\left\|S^{-1}\right\|_{2}\left\|\mathcal{E}^{n}\right\| \leq d_{n+1}\left(k^{p}+h^{r-d}\right) .
$$

Therefore (4.15) holds for $n+1$, and so it holds for $n=0, \ldots, N-q+1$. Hence, from (4.16), we obtain

$$
\left\|Y^{n}\right\| \leq \widetilde{D}\left(k^{p}+h^{r-d}\right), \quad n=0, \ldots, N-q+1 .
$$

If we now choose $X=T_{h} Y^{n+1}$ in the error equation (4.13), in view of the symmetry of the bilinear form $a,(2.3),(2.4)$ and the linearity of $T_{h}$, we have

$$
\begin{gathered}
\left(Y^{n+1}, Y^{n+1}\right)=\left(\mathcal{L} Y^{n}, Y^{n+1}\right)+k\left(S^{-1} T_{h} \Gamma_{n} e, Y^{n+1}\right)+k \alpha_{q}^{-1}\left(T_{h} \widetilde{\mathcal{E}^{n}}, Y^{n+1}\right) . \\
10
\end{gathered}
$$


Using now (4.18), (2.9), (4.20), and (4.9) in the previous relation, we obtain

$$
\begin{aligned}
\left|Y^{n+1}\right| & \leq\left(1+\max _{j}\left|\beta_{j}^{\prime}\right|\left\|S^{-1}\right\|_{2} L k\right)\left|Y^{n}\right|+\max _{j}\left|\beta_{j}^{\prime}\right|\left\|S^{-1}\right\|_{2} L k h^{d}\left\|Y^{n}\right\|+k \alpha_{q}^{-1}\left|T_{h} \widetilde{\mathcal{E}^{n}}\right| \\
& \leq(1+\widetilde{L} k)\left|Y^{n}\right|+C k\left(k^{p}+h^{r}\right), \quad n=0, \ldots N-q .
\end{aligned}
$$

Next, from (4.21), (4.14) and the discrete Gronwall lemma, we get

$$
\max _{0 \leq n \leq N-q+1}\left|Y^{n}\right| \leq C\left(k^{p}+h^{r}\right)
$$

The estimate (4.11) follows immediately from (4.22), (4.20) and (3.8).

\section{Applications}

In this section we will apply Theorem 4.1 to some specific equations, namely the Rosenau equation in $\mathbb{R}^{m}, m \leq 3$, the periodic initial value problem for a generalized Sobolev type equation in one dimension, a pseudoparabolic equation in $\mathbb{R}^{m}, m=2,3$, and to a Boussinesq system of equations.

5a. The Rosenau equation. We consider the following initial and boundary value problem for the Rosenau equation: For $t^{\star}>0$ we seek a real-valued function $u$, defined in $\bar{\Omega} \times J$, satisfying

$$
\begin{aligned}
u_{t}+\Delta^{2} u_{t} & =\nabla \cdot f(u),, & & \text { in } \Omega \times J \\
u=\frac{\partial u}{\partial n} & =0, & & \text { on } \partial \Omega \times J \\
u(\cdot, 0) & =u^{0}, & & \text { in } \Omega,
\end{aligned}
$$

where $\Omega$ is a bounded convex domain in $\mathbb{R}^{m}, m \leq 3, u^{0}$ a given smooth function and $f: \mathbb{R} \rightarrow \mathbb{R}^{m}$ a smooth vector function.

Rosenau, in [14], introduced this equation for $m=1$ and $f(x)=x+x^{2}$, to model the dynamics of large discrete systems in order to avoid the disadvantages of the Korteweg-de Vries (KdV) equation. Park, in [13], proved existence, uniqueness and reqularity results for the solution $u$ of (5a.1). Also Chung and Ha, in [6], discretize (5a.1) using finite elements in space and the backward Euler or the Crank-Nicolson method in time.

For $s \in \mathbb{N}_{0}$, denote $H^{s}$ the Sobolev space $H^{s}(\Omega), H_{0}^{s}$ the Sobolev space $H_{0}^{s}(\Omega),\|\cdot\|_{s}$ the norm in $H^{s},(\cdot, \cdot)$ the inner product in $H:=L^{2}(\Omega)=H^{0}$ and $\|\cdot\|_{0}$ the $L^{2}$-norm. Let $A: H^{4} \cap H_{0}^{2} \rightarrow H$ be defined by $A v=\left(I+\Delta^{2}\right) v$. Then $V:=D\left(A^{1 / 2}\right)=H_{0}^{2}$ and the norm in $V$ is given by $\|v\|=\left(\|v\|_{0}^{2}+\|\Delta v\|_{0}^{2}\right)^{1 / 2}$. Let $B: V \rightarrow H$ be given by $B(v)=\nabla \cdot f(v)$.

For the space discretization, consider a family $\left\{V_{h}\right\}_{0<h \leq 1}$ of finite dimensional subspaces of $H_{0}^{2}$. We assume that (having however in mind that in the case of a curved boundary $\partial \Omega$ the assumption $V_{h} \subset V$ may not hold, cf. Remark 2.1) $V_{h}$ satisfies the following approximation property

$$
\inf _{\chi \in V_{h}} \sum_{j=0}^{2} h^{j}\|v-\chi\|_{j} \leq C h^{s}\|v\|_{s}, \quad \forall v \in H^{s} \cap V, \quad 2 \leq s \leq r .
$$


In the case $m=2$, an example of finite dimensional subspaces of $H^{2}$ satisfying (5a.2) are the $C^{1}$ piecewise polynomials of degree $r-1, r \geq 4$, see [7]. We define the bilinear form $a(\cdot, \cdot)$ by

$$
a(v, w)=(v, w)+(\Delta v, \Delta w), \quad \forall v, w \in V .
$$

Then, using standard arguments, we can see that the "elliptic" projection $R_{h}: V \rightarrow V_{h}$, defined in (2.5), satisfies hypotheses (H1) and (H2), with $d=2$. Next, denote $\|\cdot\|_{L^{\infty}}$ the norm of $L^{\infty}(\Omega)$. According to Sobolev's inequality, there exists a constant $C_{\star}$, such that

$$
\|v\|_{L^{\infty}} \leq C_{\star}\|v\|, \quad \forall v \in H_{0}^{2} .
$$

Define the set $M$ by $M=\{v \in V: \exists t \in J,\|u(\cdot, t)-v\|<1\}$. Let now $\widetilde{M}=\{U \in \mathbb{R}: \exists(x, t) \in$ $\left.\Omega \times J,|u(x, t)-U|<C_{\star}\right\}$, with $C_{\star}$ the constant in (5a.3). Obviously, $f$ restricted to $\widetilde{M}$ satisfies a Lipschitz condition, with constant $L$. Thus, one can show that for $v, w \in M$ and $\varphi \in V$,

$$
(B(v)-B(w), \varphi) \leq\left(\sum_{i=1}^{m}\left\|f_{i}(v)-f_{i}(w)\right\|_{0}\right)\|\varphi\| \leq 3 L\|v-w\|_{0}\|\varphi\| .
$$

Combining the above estimate with (2.2) we obtain, for $v, w \in M$,

$$
\|B(v)-B(w)\|_{\star} \leq L_{1}\|v-w\| \quad \text { and } \quad\|T B(v)-T B(w)\|_{0} \leq L_{2}\|v-w\|_{0} .
$$

Then, it is easily seen that hypotheses (H3) and (H4) are satisfied.

Let now $U^{0}, \ldots, U^{q-1} \in V_{h}$ be approximations to $u^{0}, \ldots, u^{q-1}$, such that

$$
\sum_{j=0}^{q-1}\left\|U^{j}-u^{j}\right\|_{0} \leq C\left(k^{p}+h^{r}\right) \text { and } \sum_{j=0}^{q-1}\left\|U^{j}-u^{j}\right\| \leq C\left(k^{p}+h^{r-2}\right) .
$$

Define approximations $U^{n} \in V_{h}$ to $u^{n}, n=q, \ldots, N$, inductively by the scheme

$$
\sum_{j=0}^{q} \alpha_{j}\left[\left(U^{n+j}, \chi\right)+\left(\Delta U^{n+j}, \Delta \chi\right)\right]=k \sum_{j=0}^{q-1} \beta_{j}\left(\nabla \cdot f\left(U^{n+j}\right), \chi\right), \quad \forall \chi \in V_{h},
$$

for $n=0, \ldots, N-q$. Then from Theorem 4.1, for $k$ and $h$ sufficiently small, we have

$$
\max _{0 \leq n \leq N}\left\|u^{n}-U^{n}\right\|_{0} \leq C\left(k^{p}+h^{r}\right) \text { and } \max _{0 \leq n \leq N}\left\|u^{n}-U^{n}\right\| \leq C\left(k^{p}+h^{r-2}\right),
$$

with a constant $C\left(\|u\|_{r},\left\|u^{(p+1)}\right\|_{r}\right)$, independent of $k$ and $h$.

5b. A generalized Sobolev type equation. We consider the following periodic initial value problem for a Sobolev type equation: For $t^{\star}>0$ find a real-valued function $u$, defined in $\mathbb{R} \times J$, satisfying

$$
\begin{aligned}
-\left(b(x) u_{t x}\right)_{x}+c(x) u_{t} & =-\left(\gamma(x, t, u) u_{x}\right)_{x}+\delta(x, t, u) u_{x}+\varepsilon(x, t, u), & & \text { for } x \in \mathbb{R}, t \in J, \\
u(x, 0) & =u^{0}(x), & & \text { for } x \in \mathbb{R}, \\
u(x+1, t) & =u(x, t), & & \text { for }(x, t) \in \mathbb{R} \times J,
\end{aligned}
$$


with $b$ and $c$ strictly positive, continuously differentiable functions of $x, 1$-periodic, $\gamma, \delta$ and $\varepsilon$ continuously differentiable functions of $x, t$ and $u, 1$-periodic with respect to $x$.

Equations of the form (5b.1) have been used to model many physical phenomena (see references in [9]). An example is the equation

$$
-u_{x x t}+u_{t}+(1+u) u_{x}-\nu u_{x x}=0, \quad \nu \geq 0,
$$

which has been widely studied by Benjamin, Bona and Mahony, in [3], and other researchers, (see references in [2]), as an alternative to the Korteweg-de Vries equation, to model long unidirectional dispersive waves. The numerical solution of problems of form (5b.1) has been studied by many researchers, namely Ewing, Ford, Ting, (see also the references in [2]), using finite element methods as well as finite differences.

Define 1-periodic Sobolev spaces by $H_{\mathrm{p}}^{s}=\left\{f \in H_{\mathrm{loc}}^{s}(\mathbb{R}) / f(x+1)=f(x), x \in \mathbb{R}\right\}$, and denote $\|f\|_{s}=\|f\|_{H^{s}(I)}$ the respective norm in $H_{\mathrm{p}}^{s}$, where $I$ is an interval of length one. Also denote $(\cdot, \cdot)$ the inner product in $H:=L_{\mathrm{p}}^{2}=H_{\mathrm{p}}^{0}$ and $\|\cdot\|_{0}$ the 1 -periodic $L^{2}-$ norm.

Let $A: H_{\mathrm{p}}^{2} \rightarrow H$ be defined by $A v=-\left(b v^{\prime}\right)^{\prime}+c v$. Thus, $V=D\left(A^{1 / 2}\right)=H_{\mathrm{p}}^{1}$ with norm $\|v\|=\left(\left(b v^{\prime}, v^{\prime}\right)+(c v, v)\right)^{1 / 2}$, which is equivalent to $\|\cdot\|_{1}$. Also let $B: J \times H_{\mathrm{p}}^{2} \rightarrow H$ be given by $B(t, v(x))=-\left(\gamma(x, t, v(x)) v^{\prime}\right)_{x}+\delta(x, t, v) v^{\prime}+\varepsilon(x, t, v)$. For the space discretization consider a quasiuniform partition of $[0,1], 0=x_{0}<x_{1}<\cdots<x_{J}=1$, where $h:=\max _{j}\left(x_{j+1}-x_{j}\right)$. Let $x_{j J+i}:=j+x_{i}, j \in \mathbb{Z}, i=0, \ldots, J-1$. We extend this partition periodically to a partition of $\mathbb{R}$. For integer $r \geq 2$, let $V_{h}$ denote a space of at least continuous, 1-periodic splines, of degree $r-1$. It is well known, cf. e.g. [15, Theorem 8.12], that the family $\left\{V_{h}\right\}_{0<h \leq 1}$ satisfies the approximation property

$$
\inf _{\chi \in V_{h}} \sum_{j=0}^{1} h^{j}\|v-\chi\|_{j} \leq C h^{s}\|v\|_{s}, \quad \forall v \in H_{\mathrm{p}}^{s}, \quad 1 \leq s \leq r .
$$

We define the bilinear form $a(\cdot, \cdot)$ by

$$
a(v, w)=\left(b v^{\prime}, w^{\prime}\right)+(c v, w), \quad \forall v, w \in V .
$$

Then, we can easily see that the "elliptic" projection $R_{h}: V \rightarrow V_{h}$, defined in (2.5), satisfies hypotheses (H1) and (H2), with $d=1$. Next, denote $\|\cdot\|_{L^{\infty}}$ the norm of $L^{\infty}(\mathbb{R})$. It is easily seen that

$$
\|v\|_{L^{\infty}} \leq\|v\|_{1}, \quad \forall v \in H_{\mathrm{p}}^{1} .
$$

Because norms $\|\cdot\|_{1}$ and $\|\cdot\|$ are equivalent, define the set $M$ we introduced in $\S 2$ by, $M=$ $\left\{v \in V: \exists t \in J,\|u(\cdot, t)-v\|_{1}<1\right\}$. Consider now the set $\widetilde{M}=\{U \in \mathbb{R}: \exists(x, t) \in \mathbb{R} \times$ $J,|u(x, t)-U|<1\}$. Obviously, every real function $f \in C^{1}(\mathbb{R} \times J \times \mathbb{R})$, periodic with respect to its first variable, restricted to $\mathbb{R} \times J \times \widetilde{M}$ satisfies a Lipschitz condition with respect to its third variable, uniformly to the other two variables, with Lipschitz constant $L_{f}$. It is easily seen that

$$
\|f(\cdot, t, v)-f(\cdot, t, w)\|_{0} \leq L_{f}\|v-w\|_{0}, \quad \forall t \in J, \forall v, w \in M
$$

Thus, using (5b.4) for $\gamma, \delta, \varepsilon$, and the fact that $\gamma$ and $\delta$ are bounded restricted to $\mathbb{R} \times J \times \widetilde{M}$, one can see that for $v, w \in M$ and $\varphi \in H_{\mathrm{p}}^{1}$,

$$
(B(t, v)-B(t, w), \varphi) \leq C\left(1+\left\|w^{\prime}\right\|_{L^{\infty}}\right)\|v-w\|_{1}\|\varphi\|_{1},
$$


with a constant $C$ independent of $v, w$ and $\varphi$. Next, denote by $\|\cdot\|_{1, \infty}$ the norm of the Sobolev space $W^{1, \infty}(\mathbb{R})$. According to Sobolev's inequality, there exists a constant $C$, such that

$$
\|u\|_{1, \infty} \leq C\|u\|_{2}, \quad \forall u \in H_{\mathrm{p}}^{2}
$$

Also there exists a uniquely defined element $Q_{h} v \in V_{h}$, see, e.g., [15, Theorem 8.12], such that

$$
\left\|Q_{h} u-u\right\|_{1} \leq C h^{s-1}\|u\|_{s}, \quad 1 \leq s \quad \text { and } \quad\left\|Q_{h} u\right\|_{1, \infty} \leq C\|u\|_{1, \infty} .
$$

Since we have assumed a quasiuniform partition of $[0,1]$, the following inverse inequality holds, cf., e.g., [5, Chapter 4],

$$
\|\chi\|_{1, \infty} \leq C h^{-1 / 2}\|\chi\|_{1}, \quad \forall \chi \in V_{h} .
$$

Then, from (5b.6)-(5b.8), (5b.2) and the fact that $r \geq 2$, we can easily get

$$
\left\|R_{h} u\right\|_{1, \infty} \leq\left\|Q_{h} u\right\|_{1, \infty}+\left\|Q_{h} u-R_{h} u\right\|_{1, \infty} \leq C\left(\|u\|_{2}+h^{r-1 / 2}\|u\|_{r}\right) \leq C\|u\|_{r} .
$$

Combining the above estimate with (5b.5) we see that hypothesis (H3) is satisfied. In the sequel, we will show that (H4) is also satisfied. To this end we consider the auxiliary function $q: \mathbb{R} \times J \times \mathbb{R} \rightarrow \mathbb{R}, q(x, t, y)=\frac{1}{b(x)} \int_{0}^{y} \gamma(x, t, z) d z$. It is easily seen that $q(\cdot, t, v) \in H_{\mathrm{p}}^{1}$, for all $t \in J$, and $v \in M$. Next, for $t \in J$ and $v, w \in M$, we have

$$
a(q(\cdot, t, v), w)=\left(\gamma(x, t, v) v^{\prime}+\int_{0}^{v} \gamma_{x}(\cdot, t, z) d z-b^{\prime}(x) q(\cdot, t, v), w^{\prime}\right)+(c q(\cdot, t, v), w) .
$$

Let us define the operator $F: J \times V \rightarrow V^{\prime}$ by

$$
(F(t, v), w)=\left(\int_{0}^{v} \gamma_{x}(\cdot, t, z) d z-b^{\prime}(x) q(\cdot, t, v), w^{\prime}\right)+(c q(\cdot, t, v), w), \quad \forall w \in H_{\mathrm{p}}^{1}
$$

Then, in view of (5b.9), we have, for $v \in M$ and $w \in H_{\mathrm{p}}^{1}$,

$$
(T B(t, v), w)=(q(\cdot, t, v), w)-(T F(t, v), w)+\left(T\left(\delta(\cdot, t, v) v^{\prime}+\varepsilon(\cdot, t, v)\right), w\right) .
$$

Consider also the auxiliary functions $f_{1}, f_{2}: \mathbb{R} \times J \times \mathbb{R} \rightarrow \mathbb{R}$ defined by $f_{1}(x, t, y)=\int_{0}^{y} \gamma_{x}(x, t, z) d z$ $-b^{\prime}(x) q(x, t, y)$ and $f_{2}(x, t, y)=c(x) q(x, t, y)$. Obviously, $f_{1}$ and $f_{2}$ are continuously differentiable functions. According to the definition of $f_{1}$ and $f_{2},(5 \mathrm{~b} .4)$ and (5b.10), it is easily seen that

$$
\|F(t, v)-F(t, w)\|_{\star} \leq C\|v-w\|_{0}, \quad \forall v, w \in M
$$

Next, consider the functions $g_{1}, g_{2}: \mathbb{R} \times J \times \mathbb{R} \rightarrow \mathbb{R}$ defined by $g_{1}(x, t, y)=\int_{0}^{y} \delta(x, t, z) d z$ and $g_{2}(x, t, y)=\int_{0}^{y} \delta_{x}(x, t, z) d z$. Obviously, $\left(g_{1}(x, t, v(x))\right)_{x}-g_{2}(x, t, v(x))=\delta(x, t, v) v^{\prime}(x)$. Using now (5b.4) for $g_{1}$ and $g_{2}$, we can show

$$
\left\|\delta(\cdot, t, v) v^{\prime}-\delta(\cdot, t, w) w^{\prime}\right\|_{\star} \leq C\|v-w\|_{0}, \quad \forall v, w \in M .
$$


Using also (5b.4) for $q$ and $\varepsilon$, and (5b.11)-(5b.13), one can prove

$$
\|T B(t, v)-T B(t, w)\|_{0} \leq C\|v-w\|_{0}, \quad \forall v, w \in M .
$$

Thus hypopothesis (H4) is satisfied.

Let now $U^{0}, \ldots, U^{q-1} \in V_{h}$ be approximations to $u^{0}, \ldots, u^{q-1}$, such that

$$
\sum_{j=0}^{q-1}\left\|U^{j}-u^{j}\right\|_{0} \leq C\left(k^{p}+h^{r}\right) \quad \text { and } \quad \sum_{j=0}^{q-1}\left\|U^{j}-u^{j}\right\|_{1} \leq C\left(k^{p}+h^{r-1}\right) .
$$

Define approximations $U^{n} \in V_{h}$, to $u^{n}, n=q, \ldots, N$, inductively by the scheme

$$
\begin{aligned}
& \sum_{j=0}^{q} \alpha_{j}\left[\left(c U^{n+j}, \chi\right)+\left(b\left(U^{n+j}\right)^{\prime}, \chi^{\prime}\right)\right]=k \sum_{j=0}^{q-1} \beta_{j}\left[\left(\gamma\left(\cdot, t^{n+j}, U^{n+j}\right)\left(U^{n+j}\right)^{\prime}, \chi^{\prime}\right)\right. \\
& \left.+\left(\delta\left(\cdot, t^{n+j}, U^{n+j}\right)\left(U^{n+j}\right)^{\prime}+\varepsilon\left(\cdot, t^{n+j}, U^{n+j}\right), \chi\right)\right], \quad \forall \chi \in V_{h},
\end{aligned}
$$

for $n=0, \ldots, N-q$. Then, from Theorem 4.1, for $k$ and $h$ sufficiently small, we have

$$
\max _{0 \leq n \leq N}\left\|u^{n}-U^{n}\right\|_{0} \leq C\left(k^{p}+h^{r}\right) \text { and } \max _{0 \leq n \leq N}\left\|u^{n}-U^{n}\right\|_{1} \leq C\left(k^{p}+h^{r-1}\right),
$$

with a constant $C\left(\|u\|_{r},\left\|u^{(p+1)}\right\|_{r}\right)$, independent of $k$ and $h$.

5c. A pseudoparabolic equation. Consider now the following initial and boundary value problem: For $t^{\star}>0$ we seek a real-valued function $u$, defined in $\bar{\Omega} \times J$, such that

$$
\begin{aligned}
u_{t}(x, t)-\eta \Delta u_{t}(x, t) & =\lambda \Delta u(x, t), & & \text { in } \Omega \times J, \\
u(\cdot, 0) & =u^{0}, & & \text { in } \Omega, \\
u & =0, & & \text { on } \partial \Omega \times J,
\end{aligned}
$$

with $u^{0}$ a given smooth function, $\Omega$ a bounded convex domain, $\eta>0$ and $\lambda \in \mathbb{R}$.

Equations of this form are called pseudoparabolic, because the solution of the parabolic problem $(\eta=0$ and $\lambda>0)$, can be approximated by a sequence of solutions $u_{n}$ of problems of the form (5c.1), with $\eta=\eta_{n}$, where $\eta_{n} \rightarrow 0$, (see [16], [10] and their references). Showalter and Ting, in [16], show existence and regularity of the solution $u$ of (5c.1) and mention specific physical phenomena described by this equation.

Denote $H^{s}$ the Sobolev space $H^{s}(\Omega), H_{0}^{s}$ the space $H_{0}^{s}(\Omega)$, and $\|\cdot\|_{s}$ the usual norm in $H^{s}$. Let $H=L^{2}(\Omega)=H^{0}$ and denote $(\cdot, \cdot)$ the usual inner product in $L^{2}$ and $\|\cdot\|_{0}$ the induced norm. Also let $A: H^{2} \cap H_{0}^{1} \rightarrow L^{2}$ and $B: H^{2} \cap H_{0}^{1} \rightarrow L^{2}$ defined by $A v=(I-\eta \Delta) v$ and $B v=\lambda \Delta v$, respectively. Let now $V=D\left(A^{1 / 2}\right)=H_{0}^{1}$ with norm $\|v\|=\left(\|v\|_{0}^{2}+\eta\|\nabla v\|_{0}^{2}\right)^{1 / 2}$, which is equivalent to $\|\cdot\|_{1}$.

For the space discretization consider a triangulation of $\Omega$. For simplicity, let $V_{h}$ be the subspace of $H_{0}^{1}$ consisting of piecewise polynomials of degree at most $r-1, r \geq 2$, on the given triangulation. We assume that (having however in mind that in the case of a curved boundary $\partial \Omega$ the assumption $V_{h} \subset V$ may not hold, cf. Remark 2.1) $V_{h}$ satisfies the following approximation property

$$
\inf _{\chi \in V_{h}}\left(\|v-\chi\|_{0}+h\|v-\chi\|_{1}\right) \leq C h^{s}\|v\|_{s}, \quad \forall v \in H^{s} \cap H_{0}^{1}, \quad 1 \leq s \leq r .
$$


We define the bilinear form $a(\cdot, \cdot)$ by

$$
a(v, w)=(v, w)+\eta(\nabla v, \nabla w), \quad \forall v, w \in V .
$$

Then, we can easily see that the "elliptic" projection $R_{h}: V \rightarrow V_{h}$, defined in (2.5), satisfies hypotheses (H1) and (H2), with $d=1$. Further, we can easily see that hypotheses (H3) and (H4) are also satisfied.

Let now $U^{0}, \ldots, U^{q-1} \in V_{h}$ be approximations to $u^{0}, \ldots, u^{q-1}$, such that

$$
\sum_{j=0}^{q-1}\left\|U^{j}-u^{j}\right\|_{0} \leq C\left(k^{p}+h^{r}\right) \quad \text { and } \quad \sum_{j=0}^{q-1}\left\|U^{j}-u^{j}\right\|_{1} \leq C\left(k^{p}+h^{r-1}\right) .
$$

Define approximations $U^{n} \in V_{h}$ to $u^{n}, n=q, \ldots, N$, inductively by the scheme

$$
\sum_{j=0}^{q} \alpha_{j}\left[\left(U^{n+j}, \chi\right)+\eta\left(\nabla U^{n+j}, \nabla \chi\right)\right]=k \lambda \sum_{j=0}^{q-1} \beta_{j}\left(\nabla U^{n+j}, \nabla \chi\right), \quad \forall \chi \in V_{h},
$$

for $n=0, \ldots, N-q$. Then from Theorem 4.1, for $k$ and $h$ sufficiently small, we have

$$
\max _{0 \leq n \leq N}\left\|u^{n}-U^{n}\right\|_{0} \leq C\left(k^{p}+h^{r}\right) \quad \text { and } \max _{0 \leq n \leq N}\left\|u^{n}-U^{n}\right\|_{1} \leq C\left(k^{p}+h^{r-1}\right),
$$

with a constant $C\left(\|u\|_{r},\left\|u^{(p+1)}\right\|_{r}\right)$, independent of $k$ and $h$.

5d. A system of Boussinesq type. Finally we consider the following system of initial and boundary value problem of Boussinesq type. Given $t^{\star}>0$ find $\underline{u}=\left(u_{1}, u_{2}\right):\left[0, L_{0}\right] \times J \rightarrow \mathbb{R}^{2}$, $L_{0}>0$, such that

$$
\begin{aligned}
\underline{u}_{t}-\frac{1}{6} \underline{u}_{x x t} & =B(\underline{u}), & & \text { in }\left[0, L_{0}\right] \times J \\
\underline{u}(\cdot, 0) & =\underline{u}^{0}, & & \text { in }\left[0, L_{0}\right], \\
\underline{u} & =\underline{0}, & & \text { in }\left\{0, L_{0}\right\} \times J,
\end{aligned}
$$

with $\underline{u}^{0}$ a given sufficiently smooth vector function and $B:\left[0, L_{0}\right] \times J \rightarrow \mathbb{R}^{2}$ defined by $B(\underline{v})=$ $\left(-\left(v_{2}\right)_{x}-\left(v_{1} v_{2}\right)_{x},-\left(v_{1}\right)_{x}-v_{2}\left(v_{2}\right)_{x}\right)$. Also for $v_{1}$ and $v_{2}$ real-valued differentiable functions in $\left[0, L_{0}\right] \times J$, we denote $\left(\left(v_{1}\right)_{x},\left(v_{2}\right)_{x}\right)$ by $\underline{v}_{x}$ and $\left(\left(v_{1}\right)_{t},\left(v_{2}\right)_{t}\right)$ by $\underline{v}_{t}$.

System (5d.1) is an approximation of the two-dimensional propagation of surface waves in a uniform horizontal channel of length $L_{0}$, filled with an irrotational, incompressible invisid liquid which in its undisturbed state has depth 1, (cf. [4]). Also in this report, Bona and Chen prove existence, uniqueness and regularity results for the solution $\underline{u}$ of (5d.1).

For $s \in \mathbb{N}$, denote $H^{s}$ the Sobolev space $H^{s}\left(0, L_{0}\right), H_{0}^{s}$ the Sobolev space $H_{0}^{s}\left(0, L_{0}\right)$ and $|\cdot|_{s}$ the usual norm in $H^{s}$. Next, let $\widetilde{H^{s}}:=H^{s} \times H^{s}, \widetilde{H_{0}^{s}}:=H_{0}^{s} \times H_{0}^{s}, H=\widetilde{H^{0}}$ and consider the following norm in $\widetilde{H^{s}},\|\underline{v}\|_{s}=\left(\left|v_{1}\right|_{s}^{2}+\left|v_{2}\right|_{s}^{2}\right)^{1 / 2}$. Also denote by $<\cdot, \cdot>$ the usual inner product in $H^{0}$ and by $(\underline{v}, \underline{w})=<v_{1}, w_{1}>+<v_{2}, w_{2}>$ the inner product in $H$. Consider now the operators $\partial_{x}: \widetilde{H^{1}} \rightarrow \widetilde{H^{0}}$ and $A: \widetilde{H^{2}} \cap \widetilde{H_{0}^{1}} \rightarrow H$, defined by $\partial_{x} \underline{v}=\left(\left(v_{1}\right)_{x},\left(v_{2}\right)_{x}\right)$ and $A \underline{v}=\left(I-\frac{1}{6} \partial_{x}^{2}\right) \underline{v}$, respectively. Then $V=D\left(A^{1 / 2}\right)=\widetilde{H_{0}^{1}}$ with norm $\|\underline{v}\|=\left(\|\underline{v}\|_{0}^{2}+\frac{1}{6}\left\|\partial_{x} \underline{v}\right\|_{0}^{2}\right)^{1 / 2}$, which is equivalent to $\|\cdot\|_{1}$. 
For the space discretization, consider a partition of $\left[0, L_{0}\right], 0=x_{0}<x_{1}<\cdots<x_{J}=L_{0}$, where $h:=\max _{0 \leq j \leq J}\left(x_{j+1}-x_{j}\right)$ and, for integer $r \geq 2$, let $S_{h}=\left\{\chi \in C\left[0, L_{0}\right]: \chi_{\left.\right|_{\left[x_{j}, x_{j+1}\right]}}\right.$ polynomial of degree $\left.r-1, j=0, \ldots, J-1, \chi(0)=\chi\left(L_{0}\right)=0\right\}$. We can easily see that the family $\left\{V_{h}\right\}_{0<h \leq 1}, V_{h}=S_{h} \times S_{h}$, satisfies the approximation property

$$
\inf _{\underline{\chi} \in V_{h}} \sum_{j=0}^{1} h^{j}\|\underline{v}-\underline{\chi}\|_{j} \leq C h^{s}\|\underline{v}\|_{s}, \quad \forall \underline{v} \in \widetilde{H^{s}} \cap \widetilde{H_{0}^{1}}, \quad 1 \leq s \leq r .
$$

We define the bilinear form $a(\cdot, \cdot)$ by

$$
a(\underline{v}, \underline{w})=(\underline{v}, \underline{w})+\frac{1}{6}\left(\partial_{x} \underline{v}, \partial_{x} \underline{w}\right), \quad \forall \underline{v}, \underline{w} \in V .
$$

Then, we can easily see that the "elliptic" projection $R_{h}: V \rightarrow V_{h}$, defined in (2.5), satisfies hypotheses (H1) and (H2), with $d=1$. Also, it can be seen that hypotheses (H3) and (H4) are also satisfied.

Let now $\underline{U}^{0}, \ldots, \underline{U}^{q-1} \in V_{h}$ be approximations to $\underline{u}^{0}, \ldots, \underline{u}^{q-1}$, such that

$$
\sum_{j=0}^{q-1}\left\|\underline{U}^{j}-\underline{u}^{j}\right\|_{0} \leq C\left(k^{p}+h^{r}\right) \quad \text { and } \quad \sum_{j=0}^{q-1}\left\|\underline{U}^{j}-\underline{u}^{j}\right\|_{1} \leq C\left(k^{p}+h^{r-1}\right) .
$$

Define approximations $\underline{U}^{n} \in V_{h}$ to $u^{n}, n=q, \ldots, N$, inductively by the scheme

$$
\sum_{j=0}^{q} \alpha_{j}\left[\left(\underline{U}^{n+j}, \underline{\chi}\right)+\frac{1}{6}\left(\partial_{x} \underline{U}^{n+j}, \partial_{x} \underline{\chi}\right)\right]=k \sum_{j=0}^{q-1} \beta_{j}\left(B\left(\underline{U}^{n+j}\right), \underline{\chi}\right), \quad \forall \chi \in V_{h},
$$

for $n=0, \ldots, N-q$. Then using Theorem 4.1, for $k$ and $h$ sufficiently small, we have

$$
\max _{0 \leq n \leq N}\left\|\underline{u}^{n}-\underline{U}^{n}\right\|_{0} \leq C\left(k^{p}+h^{r}\right) \quad \text { and } \quad \max _{0 \leq n \leq N}\left\|\underline{u}^{n}-\underline{U}^{n}\right\|_{1} \leq C\left(k^{p}+h^{r-1}\right),
$$

with a constant $C\left(\|u\|_{r},\left\|u^{(p+1)}\right\|_{r}\right)$, independent of $k$ and $h$.

Acknowledgement. The author would like to thank his adviser Professor Georgios D. Akrivis for suggesting the problem and contributing several valuable remarks.

\section{REFERENCES}

1. Akrivis, G., Crouzeix, M., Makridakis, Ch., Implicit-explicit multistep finite element methods for nonlinear parabolic problems, Math. Comp. To appear.

2. Arnold, D.N., Douglas, J.Jr., Thomée, V., Superconvergence of a finite element approximation to the solution of a Sobolev equation in a single space variable, Math. Comp. 36 (1981), 53-63.

3. Benjamin, T.B., Bona, J.L., Mahony, J.J., Model equations for long waves in nonlinear dispersive systems, Philos. Trans. Roy. Soc. London Ser. A 272 (1972), 47-78.

4. Bona, J.L., Chen, M., A Boussinesq system for two-way propagation of nonlinear dispersive waves, TICAM REPORT 96-36, Texas Institute for Computational and Applied Mathematics (1996). 
5. Brenner, S.C., Scott, L.R., The Mathematical Theory of Finite Element Methods, Springer-Verlag, New York, 1994.

6. Chung, S.K., Ha, S.N., Finite element Galerkin solutions for the Rosenau equation, Applicable Analysis 54 (1994), 39-56.

7. Douglas, J.Jr., Dupont, T., Percell, P., Scott, R., A family of $C^{1}$ finite elements with optimal approximation properties for various Galerkin methods for 2nd and 4th order problems, R.A.I.R.O. Numer. Anal. 13 (1979), $227-255$.

8. Dautray, R., Lions, J.-L., Mathematical Analysis and Numerical Methods for Science and Technology, Volume 3, Spectral Theory and Applications, Springer-Verlang, Berlin Heidelberg, 1990.

9. Ewing, R.E., Numerical solution of Sobolev partial differential equations, SIAM J. Numer. Anal. 12 (1975), $345-363$.

10. Ewing, R.E., The approximation of certain parabolic equations backward in time by Sobolev equations, SIAM J. Math. Anal. 6 (1975), 283-294.

11. Hairer, E., Nørsett, S.P., Wanner, G., Solving Ordinary Differential Equations I, Nonstiff Problems, Second Revised Edition, Springer-Verlag, Heidelberg, 1993.

12. Henrici, P., Discrete Variable Methods in Ordinary Differential Equations, John Wiley \& Sons, Inc., New York, 1962.

13. Park, M.A., On the Rosenau equation in multidimensional space, Nonlinear Anal. 21 (1993), 77-85.

14. Rosenau, P., Dynamics of dense discrete systems, Prog. Theoretical Phys. 79 (1988), 1028-1042.

15. Schumaker, L.L., Spline Functions: Basic Theory, Wiley, New York, 1980.

16. Showalter, R.E., Ting, T.W., Pseudoparabolic partial differential equations, SIAM J. Math. Anal. 1 (1970), $1-26$. 